\title{
Social identity and social value orientations ${ }^{\text {th }}$
}

\author{
Ozan Aksoya,b,* \\ ${ }^{a}$ Department of Quantitative Social Science, University College London, 55-59 Gordon Square, WC1H 0NU, London \\ $U K$ \\ ${ }^{b}$ Nuffield College, New Road OX1 1NF, Oxford UK
}

\begin{abstract}
This study provides an extension of the social value orientation model and a tool, other-other Decomposed Games, to quantify the influence of social identity on social value orientations. Social identity is induced experimentally using the minimal group paradigm. Subsequently, the weights subjects add to the outcomes of outgroup others relative to ingroup others and to the absolute difference between the outcomes of ingroup and outgroup others are estimated. Results are compared to a control condition in which social identity is not induced. Results show that when the outgroup is better off than the ingroup, the average subject is spiteful: they derive negative utility from the outcomes of the outgroup other. When the ougroup is worse off than the ingroup, the average subject attaches similar weights to the outcomes of outgroup and ingroup others. There is also significant variation across subjects with respect to the level of ingroup bias.
\end{abstract}

Keywords: Cooperation, social value orientation, minimal groups, social identity

\section{Introduction}

A quick glance at any major newspaper nowadays will, very likely, show that humans are willing to incur significant costs to members of outgroups in order to protect or better the outcomes for the ingroup. Children whose parents are caught crossing the border illegally are separated from their parents to deter illegal entry to the US 11 Legal and "skilled" immigrants in the UK are required to pay an annual Immigration Health Surcharge of $£ 400$, in addition to the expensive visa fees and the usual tax contributions to the National Health Service 2 Almost all countries are imposing tariffs or quotas to certain foreign goods to protect domestic producers 3 There are very strong barriers to international labor mobility, legal or illegal. All of these show that humans value the outcomes of ingroup members more than the outcomes of outgroup members. But how much more?

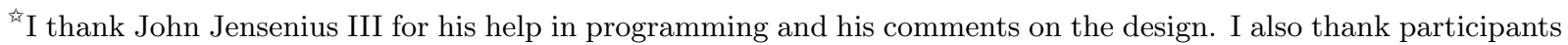
of the CESS Colloquium Series, Akitaka Matsuo, and Wojtek Przepiorka. The experiment reported in this paper was conducted at Nuffield CESS. This research is supported by the Netherlands Organization for Scientific Research (NWO) under grant 446-13-004. The Stata code and the dataset used to produce the results reported in this article are available for replication at https://osf.io/wxra3/.

* Corresponding author

Email address: ozan.aksoy@ucl.ac.uk (Ozan Aksoy)

${ }^{1}$ https://www.independent.co.uk/news/world/americas/us-politics/us-immigration-children-audio-trump-borderpatrol-separate-families-parents-detention-center-a8405501.html

${ }^{2}$ https://www.gov.uk/healthcare-immigration-application

${ }^{3}$ http://tariffdata.wto.org/Default.aspx?culture=en-US
} 
Harvard economist Dani Rodrik asks a simple and related question: "how strong a preference must we have for our fellow citizens relative to foreigners to justify the existing level of barriers on international labor mobility" (p13 Rodrik, 2017). After a simple calculation based on a plausible scenario, he concludes that "we must place a weight on the utility of fellow citizens that is at least between four and five times greater than the weight we place on foreigners". Or equivalently, a foreigner must be worth less than 22 percent of a fellow citizen. In a similar exercise, Kopczuk et al. (2005) argue that the observed levels of international assistance to developing countries imply that Americans must value their fellow citizens' outcomes about six times more than they value foreign citizens' outcomes. Or equivalently, a foreign citizen is worth 17 percent of a fellow citizen. It thus appears that the weight attached to the outcomes of outgroup members is 17 to 22 percent of the weight attached to the outcomes of ingroup members. But how accurate is this estimate? The calculations of Rodrik (2017) and Kopczuk et al. (2005) are rather indirect. They use the levels of barriers on international labor mobility and foreign assistance to estimate the relative weights. Barriers to labor mobility and foreign assistance are complex policies that are influenced by many factors, in addition to how much actors weight the outcomes of outgroups.

In this paper, I propose a simple tool to estimate directly how much actors weight the outcomes of outgroup members relative to the outcomes of ingroup members. I build on the social value orientation and the minimal group paradigm literatures. The social value orientation literature investigates how actors value certain outcome allocations between self and others (Griesinger and Livingston, 1977; McClintock, 1972; Schulz and May, 1989). Cooperative orientation, maximizing the sum of the payoffs for self and others; competitive orientation, maximizing the difference between the payoffs for self and others in favor of self; equality orientation, minimizing the inequality between outcomes are some of the social value orientations distinguished in the literature. Numerous methods have been developed to measure social value orientations (e.g., Liebrand and McClintock, 1988; Kuhlman et al., 1992; Van Lange, 1999; Murphy et al., 2011; Aksoy and Weesie, 2012, 2013). All of these methods involve some form of Decomposed Games in which subjects are asked to choose a certain outcome allocation between self and others among a menu of possible self-other allocations.

There is a hidden but strong link between the social value orientation literature and the minimal group paradigm. The minimal group paradigm is about how actors value outcome allocations between two others, e.g., one ingroup and one outgroup (Tajfel et al., 1971; Tajfel, 1970). In other words, as opposed to the social value orientation literature, the minimal group paradigm involves other-other allocations instead of self-other allocations. In fact, in minimal group experiments selfother allocations are carefully avoided. This is because in minimal group experiments, subjects' own individual interests should not be at stake to isolate the influence of mere social categorization from any form of realistic conflict (e.g., Sherif et al., 1961). Because of this omission of self from outcome allocation tasks, the tools of the social value orientation literature cannot readily be applied to minimal group settings.

In this paper, I explicitly bridge the social value orientation literature with the minimal group paradigm. I extend the social value orientation model to other-other allocations (also see Macro and Weesie, 2016). Moreover, I also show that the classical self-other Decomposed Games of the social value orientation literature can easily be adapted to other-other allocations, hence to minimal group setting. Using experimental data, I quantify the influence of social identity on social value orientations. 


\section{Theory: Social Value Orientations in other-other allocations}

In the classical social value orientation model, for an outcome allocation for self $(x)$ and other $(y)$, an actor $i$ attaches a $w_{i}$ weight to the outcome of other such that (McClintock, 1972, Griesinger and Livingston, 1977; Aksoy and Weesie, 2012, 2014):

$$
U_{i}(x, y) \equiv U_{i}^{*}\left(x, y ; w_{i}\right)=x+w_{i} y .
$$

Let's now assume that there are two types of others, ingroup and outgroup. Let $I(O)$ denote the set of ingroup (outgroup) others. Consider an other-other allocation situation in which the ingroup other gets $y^{I}$, the outgroup other gets $y^{O}$, and there is no outcome for self, i.e., $x=0$. In this situation, the social value orientation model in (1) can be written as:

$$
U_{i}\left(y^{I}, y^{O}\right) \equiv U_{i}^{*}\left(y^{I}, y^{O} ; w_{i}^{I}, w_{i}^{O}\right)=w_{i}^{I} y^{I}+w_{i}^{O} y^{O}
$$

where $w_{i}^{I}$ and $w_{i}^{0}$ are the weights actors attach to the outcomes of ingroup and outgroup others, respectively. Because utility is defined up to positive affine transformations, and assuming that $w_{i}^{I}>0$, equation 2 can be written as:

$$
U_{i}\left(y^{I}, y^{O}\right) \equiv U_{i}^{*}\left(y^{I}, y^{O} ; \theta_{i}^{O}\right)=y^{I}+\theta_{i}^{O} y^{O} \quad \text { with } \quad \theta_{i}^{O}=\frac{w_{i}^{O}}{w_{i}^{I}} .
$$

Equation (3) is now equivalent to the model in equation (1) where the outcomes for self and other are replaced by the outcomes for ingroup other and outgroup other, respectively. Consequently, the weight actors attach to the outcomes of outgroup others relative to ingroup others can easily be estimated using other-other Decomposed Games just as the social value orientations are estimated with self-other Decomposed Games (e.g., as in Aksoy and Weesie, 2012).

Finally, social value orientation research has shown that some people also consider inequality in outcomes, such as those with equality or maximin orientations (Schulz and May, 1989; Grzelak et al., 1977; Aksoy and Weesie, 2012; Macro and Weesie, 2016). These orientations are typically captured by adding another term in equation (1), the absolute inequality between the outcomes for self and other. In the other-other allocation case, an equivalent term will be adding the absolute inequality between the outcomes for ingroup and outgroup others. Thus $4^{4}$

$$
U_{i}\left(y^{I}, y^{O}\right) \equiv U_{i}^{*}\left(y^{I}, y^{O} ; \theta_{i}^{O}, \beta_{i}\right)=y^{I}+\theta_{i}^{O} y^{O}-\beta_{i}\left|y^{I}-y^{O}\right|
$$

A useful reinterpretation of the model in (4) is the following:

$$
U_{i}\left(y^{I}, y^{O}\right) \equiv U_{i}^{*}\left(y^{I}, y^{O} ; \theta_{i}^{O}, \beta_{i}\right)= \begin{cases}y^{I}+\frac{\theta_{i}^{O}+\beta_{i}}{1-\beta_{i}} y^{O} & \text { if } y^{I} \geq y^{O} \\ y^{I}+\frac{\theta_{i}^{O}-\beta_{i}}{1+\beta_{i}} y^{O} & \text { if } y^{I}<y^{O}\end{cases}
$$

\footnotetext{
${ }^{4}$ When inequality concerns are introduced, in other-other allocations in which self gets zero it can be argued that actors may take two additional terms into account: the difference between outcomes for ingroup others and self as well as the difference between outgroup others and self. In this case, the model can be written as $U=$ $x+w_{i}^{I} y^{I}+w_{i}^{O} y^{O}-b_{i}^{I}\left|y^{I}-x\right|-b_{i}^{O}\left|y^{O}-x\right|-\beta_{i}\left|y^{I}-y^{O}\right|$. Because in other-other allocations $x=0$, this alternative formulation can be re-arranged (assuming $y^{I}>0, y^{O}>0$, and $w_{i}^{I}>b_{i}^{I}$ ) such that $U=y^{I}+\frac{w_{i}^{O}-b_{i}^{O}}{w_{i}^{I}-b_{i}^{I}} y^{O}-\beta_{i}\left|y^{I}-y^{O}\right|$, which is equivalent to the formulation in equation $\sqrt{4}$ with $\frac{w_{i}^{O}-b_{i}^{O}}{w_{i}^{I}-b_{i}^{I}}=\theta_{i}^{O}$.
} 
Equation (4) is mathematically equivalent to (5) when $\beta<1$. My empirical results below will indeed show that $\beta<1$, hence I will use (4) and (5) interchangeably. The specification in (5) is easier to interpret than that in (4). In (5) we have two separate terms that represent the weights attached to the outcomes of the outgroup other relative to the ingroup. When the outgorup is worse off than the ingroup this weight is $\frac{\theta_{i}^{O}+\beta_{i}}{1-\beta_{i}}$. When the outgroup other is better off than the ingroup other the weight to the outgroup is $\frac{\theta_{i}^{O}-\beta_{i}}{1+\beta_{i}}$. Hence, while $\sqrt{5}$ and 44 are mathematically equivalent, specification (5) provides an alternative more convenient interpretation.

One could in principle modify the model in (4) by taking not the outcomes for the ingroup or outgroup, but the utilities for the ingroup and outgroup. For example, one could define $U_{i}^{\prime}\left(y^{I}, y^{O}\right)$ as $U_{i}^{\prime}\left(y^{I}, y^{O}\right)=U^{I}\left(y^{I}, y^{O}\right)+\theta_{i}^{\prime O} U^{O}\left(y^{I}, y^{O}\right)-\beta_{i}^{\prime}\left|U^{I}\left(y^{I}, y^{O}\right)-U^{O}\left(y^{I}, y^{O}\right)\right|$. That is, replacing the outcomes of ingroup and outgroup others with utilities for ingroup and outrgroup others. Alternatively, one could define equality as $y^{I}=\theta y^{O}$, and hence replace the term $\left|y^{I}-y^{O}\right|$ in (4) with $\left|y^{I}-\theta y^{O}\right|$. These alternative formulations are examples of interdependent utility, i.e., actors are interested in utilities of other actors not just outcomes (Becker, 1993). In this article, I don't consider interdependent utility.

\section{Method}

\subsection{Subjects}

186 subjects were recruited with the Online Recruitment System for Economic Experiments (ORSEE; Greiner (2004)). Majority of the subjects were students at the University of Oxford from a variety of different study fields. Subjects were on average 30 years old (S.D.=14) and $58 \%$ of them were female 5

\subsection{Procedure}

Subjects participated in one of ten sessions in Hilary Term (February-March) 2014. Subjects in seven sessions were assigned to the experimental group and in the remaining three sessions to the control group. Seven sessions were run between 16 and 24 subjects and three sessions were run between 12 to 14 subjects. Subjects sat randomly in one of the cubicles in the Centre for Experimental Social Sciences (CESS) lab at Nuffield College, University of Oxford. Subjects could not see each other or the experimenter during the experiment. This also meant that the subjects were not fully aware of the total number of subjects in the experiment, though they might have a rough guess about the session size (the median session size was 20). The experiment was carried out on computers using z-tree (Fischbacher, 2007).

\subsubsection{Experimental group}

After general instructions, subjects in the experimental group were shown five pairs of paintings by Wassily Kandinsky and Paul Klee. For each pair, subjects chose the painting they liked more. $50 \%$ of subjects in a session were classified as Kandinskys, and the remaining $50 \%$ as Klees, based on subjects' relative preferences. Each subject was privately informed about his/her group.

\footnotetext{
${ }^{5}$ The experiment reported here is embedded in a larger study which included additional unrelated tasks. These additional tasks were administered after the procedure described here took place and were analyzed elsewhere. See Aksoy (2015) for details of these additional tasks.
} 
After classification, a collective quiz in which subjects guessed the painters of two paintings (Klee or Kandinsky) was administered. Subjects earned $£ 0.8$ if at least $50 \%$ of their group correctly guessed the two painters. Subjects earned a further $£ 0.8$ if their group correctly answered as many questions as the other group. Quiz results were shown only after the experiment was completed.

After the collective quiz, subjects made decisions in 10 other-other Decomposed Games shown in the appendix. The order of these 10 games was varied in two factors. These 10 games were modified versions of the self-other Decomposed Games used by Aksoy and Weesie (2012) ${ }^{6}$ Recipients in these Decomposed Games were a randomly selected ingroup member and a randomly selected outgroup member. At the end of the experiment, one Decomposed Game was selected at random, and two actual other subjects received the tokens based on a subject's decision $(20$ tokens $=£ 1)$. Similarly, each subject was a recipient for a randomly selected other subject.

\subsubsection{Control group}

The control group followed the procedure above but without inducing group identity. Subjects stated their preferences in the same 5 painting pairs. However, they were not classified as Klees or Kandinskys. They completed the same guessing quiz but they were rewarded for their individual success: for each correct guess, a subject earned $£ 0.8$. Finally, subjects decided in the same 10 other-other Decomposed Games. Different from the experimental group, the two recipients were two other subjects randomly selected from the session, without any reference to any groups.

\section{Results}

I follow the estimation procedure described in Aksoy and Weesie (2012). In this procedure, the outcomes in an other-other Decomposed Game are transformed into utilities via equation (4). In addition, an additive random utility term $\epsilon$ is added to the model to have stochastic behavioral predictions. The random utility term makes the utility model statistically estimable. How much a subject $i$ prefers option A relative to option $\mathrm{B}$ is the utility difference in options $\mathrm{A}$ and $\mathrm{B}$ in a game:

$$
U_{A B}\left(y^{I}, y^{O} ; \theta_{i}^{O}, \beta_{i}\right)=\left(y_{A}^{I}-y_{B}^{I}\right)+\theta_{i}^{O}\left(y_{A}^{O}-y_{B}^{O}\right)-\beta_{i}\left(\left|y_{A}^{I}-y_{A}^{O}\right|-\left|y_{B}^{I}-y_{B}^{O}\right|\right)+\left(\epsilon_{A}-\epsilon_{B}\right)
$$

where $y_{A}^{I}$ is the outcome for ingroup other in option A and $y_{B}^{O}$ is the outcome for outgroup other in option B in a Decomposed Game. A subject prefers option A in a Decomposed Game when $U_{A B}>0$. Following Aksoy and Weesie (2012), $\left(\theta^{O}, \beta\right)$ are treated as bi-variate normally distributed variables and $\epsilon$ is assumed to have an independent normal distribution with zero mean and nonzero variance. This implies a multilevel probit model in which the dependent variable is a subject's preferences in the 10 Decomposed Games and independent variables are the outcome differences given in equation (6). The distribution of $\left(\theta^{O}, \beta\right)$, the variance of $\left(\epsilon_{A}-\epsilon_{B}\right)$, and the empirical Bayes predictions (posterior means) of $\theta^{O}$ and $\beta$ per subject are estimated with the Stata program GLLAMM (Rabe-Hesketh et al., 2002). The replication material with the Stata code and the data are available at https://osf.io/wxra3/.

\footnotetext{
${ }^{6}$ The modifications aimed to improve the statistical precision to estimate the social value orientation parameters based on the results reported in Aksoy and Weesie (2012) and additional simulations.
} 
Table 1 and Figure 1 show the results. When social identity is induced (experimental group), the estimated mean of $\theta^{O}$ is 0.2 which is significantly different from both zero and one. This means that when the inequality between ingroup and outgroup is zero, the average weight subjects add to the outcomes of outgroup others is only $20 \%$ of the weight they add to the outcomes of ingroup others. The estimated mean of $\beta$ is 0.39 and significantly different from zero (and one). This means that while subjects add a small weight to the outcomes of outgroup others relative to ingroup others, they are still concerned with reducing inequality between ingroup and outgroup others. There is also a negative correlation between $\theta^{O}$ and $\beta$.

A further noteworthy finding is the significant and large variance of $\theta^{O}$ in the experimental group. An estimated variance of 0.34 implies that although on average there is significant ingroup bias, there is also a significant variation among subjects regarding the level of ingroup bias. A minority of subjects in fact have $\theta^{O}$ values very close to, but never exceeding one (see Figure 1 -a). These subjects could be described as "multicultural" as they add very similar weights to ingroup and outgroup others' outcomes. On the other hand, quite a few subjects (about 36\%) add not only lower but negative weights to the outcomes of outgroup others, displaying a very high level of ingroup bias.

In the control group in which social identity is not induced $\theta^{O}$ is estimated as virtually 1 . This shows that without any difference in group identities, equal weights are added to the outcomes of two random others. This finding adds confidence to the estimation method because any value significantly different from 1 would hint at a methodological artifact and cast doubt on the validity of the results. Also, the difference between the means of $\theta^{O}$ in the experimental and control groups is highly significant ( $\mathrm{p} 2$-sided $<0.001$ ). In the control group, the estimated variance of $\theta^{O}$ is insignificant and the mean of $\beta$ is estimated as 0.235 . The difference in average $\beta \mathrm{s}$ in the control and experimental groups is not very large and in fact statistically marginally insignificant (p 2-sided = 0.06). Similar to the variance of $\theta$, the variance of $\beta$ in the control group is statistically insignificant. Finally, the variance of the error term $\left(\epsilon_{A}-\epsilon_{B}\right)$ is virtually identical in the experimental and control groups.

A $\theta^{0}=0.2$ is a remarkably similar estimate compared to the indirect estimates of Rodrik (2017) and Kopczuk et al. (2005). The utility weight that subjects attach to the inequality between ingroup and outgroup $(\beta)$, however, is an argument that Rodrik (2017) and Kopczuk et al. (2005) omit. The existence of the $\beta$ term somewhat complicates the interpretation of $\theta^{0}$. This is because the actual weight one attaches to the outcome of outgroup others is affected by the inequality between ingroup and outgroup. For a more convenient interpretation, I will now use the alternative and equivalent specification of the utility function given in (5). In this alternative interpretation we can define two weights attached to the outcomes of outgroup others relative to that of ingroup others when (i) the outgroup is worse off than the ingroup and (ii) the outgroup is better off than the ingroup.

These weights are obtained using the $\beta$ and $\alpha$ parameters (see equation 5). More precisely, when the outgroup is worse off than the ingroup (i.e., $y^{I}-y^{O}>0$ ) the net weight one adds to the outcomes of outgroup others relative to ingroup others is $\frac{\theta^{0}+\beta}{1-\beta}$. Substituting the estimated means of $\theta^{0}$ and $\beta$ gives us an estimate of $\frac{0.2+0.39}{1-0.39}=0.97$. In other words, when ougroup others are worse off their outcomes are worth $97 \%$ of the outcomes for the ingroup, for an average subject. This means that an average subject will be somewhat indifferent to a policy that redistributes outcomes from the ingroup to the outgroup, when the outgroup is worse off. When the outgroup is better off than the ingroup the net weight one adds to the outcomes of the outgroup relative to the ingroup 
Table 1: Social value orientation estimates for the experimental and control groups. $\theta^{O}=$ "outgroup cooperative orientation parameter"; $\beta=$ "equality orientation parameter"; $\epsilon_{A}, \epsilon_{B}=$ evaluation error. For the variances, p-values are derived from the correct boundary tests using the mixture distribution (see Self and Liang, 1987).

\begin{tabular}{|c|c|c|c|c|}
\hline \multirow[b]{2}{*}{ Paral } & \multicolumn{2}{|c|}{ Experimental Group } & \multicolumn{2}{|c|}{ Control Group } \\
\hline & Coeff. & S.E. & Coeff. & S.E. \\
\hline $\operatorname{mean}\left(\theta^{O}\right)$ & $.200^{* * *}$ & .059 & $1.003^{* * *}$ & .154 \\
\hline $\operatorname{mean}(\beta)$ & $.389^{* * *}$ & .032 & $.235^{* *}$ & .094 \\
\hline $\operatorname{var}\left(\theta^{O}\right)$ & $.327^{* * *}$ & .087 & .095 & .073 \\
\hline $\operatorname{var}(\beta)$ & $.050^{* * *}$ & .020 & .063 & .062 \\
\hline $\operatorname{cov}\left(\theta^{O}, \beta\right)$ & $-.099^{* * *}$ & .038 & -.073 & .058 \\
\hline $\operatorname{var}\left(\epsilon_{A}-\epsilon_{B}\right)$ & $.021^{* * *}$ & .002 & $.021^{* * *}$ & .005 \\
\hline $\mathrm{N}$ (Subject) & \multicolumn{2}{|c|}{146} & \multicolumn{2}{|l|}{40} \\
\hline N(Decision) & \multicolumn{2}{|c|}{1460} & \multicolumn{2}{|c|}{400} \\
\hline log-likelihood & \multicolumn{2}{|c|}{-723.762} & \multicolumn{2}{|c|}{-140.875} \\
\hline
\end{tabular}

is $\frac{\theta^{0}-\beta}{1+\beta}$. Substituting the estimated means gives us $\frac{0.2-0.39}{1+0.39}=-0.14$. This implies that when the outgroup is in a better position than the ingroup, the average subject is spiteful: they derive negative utility from the outcomes of the outgroup other. Figure1 1 b shows the scatter plot of these two weights. An interesting finding is the positive correlation between the two weights. The figure also shows that there is precisely one subject who adds a negative weight to the outcomes of the outgroup even when the outgroup is worse off than the ingroup. When the outrgoup is better off than the ingroup, the majority of subjects are spiteful toward the outgroup.

\section{Conclusions}

In this study I bridge the social value orientation literature with the minimal group paradigm. I extend the social value orientation model to other-other allocations, particularly to the case in which the two recipients are an ingroup member and an outgroup member. Moreover, I provide a set of other-other Decomposed Games. Using these games and inducing social identity via minimal groups, I estimate the weights subjects add to the outcomes of outgroup others relative to ingroup others and to the absolute difference between the outcomes of ingroup and outgroup others. I compare these results to a control condition in which social identity is not induced. This method quantifies clearly the effect of group identity on social value orientations.

Results show that the average weight subjects add to the outcomes of outgroup others relative to ingroup others is negative when the outgroup is better off than the ingroup. In other words, the average subject is spiteful toward the outrgoup when the outgroup is in an advantageous position compared with the ingroup. When the outgroup is worse off than the ingorup, the average subject weights the outcomes of ingroup and outgroup others similarly. There is also a significant variation among subjects with respect to the level of ingroup bias. While a substantial number of subjects show high levels of ingroup bias, a minority of "multicultural" subjects display little bias. This 
Figure 1: Scatter plots of utility weights in the experimental group for the two alternative interpretations of the social value orientation model.
(a) $\theta$ and $\beta$

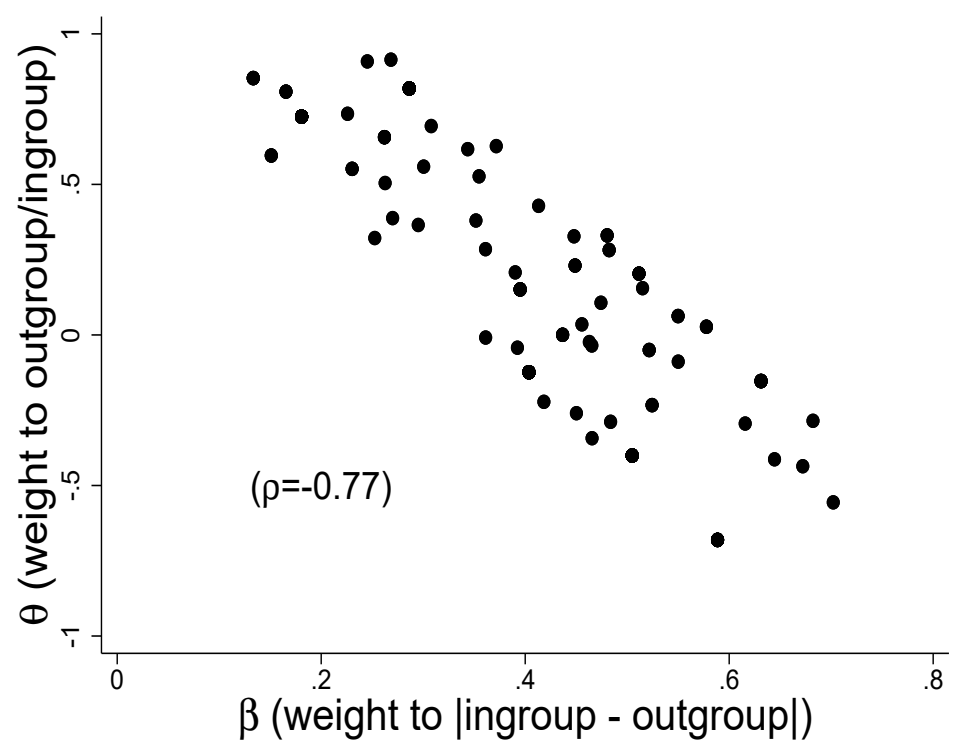

(b) $\frac{\theta^{O}+\beta}{1-\beta}$ and $\frac{\theta^{O}-\beta}{1+\beta}$

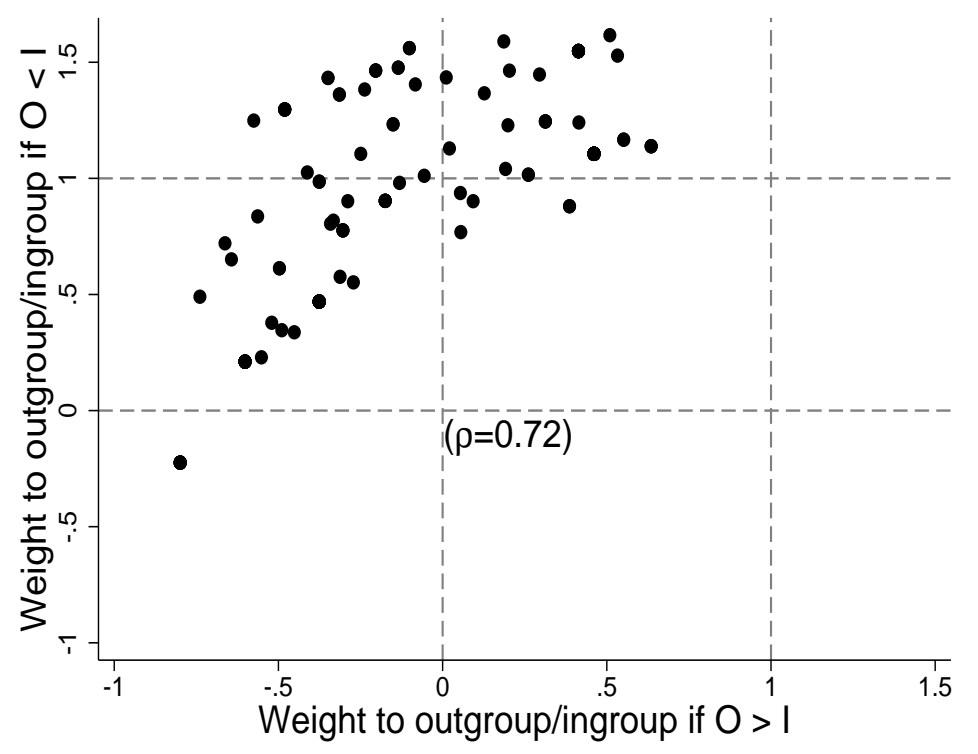


variation is an interesting finding and future research should focus on identifying subject level characteristics that explain this variation.

The method I present here is very easy to implement. The 10 other-other Decomposed Games can easily be embedded in a survey or an experimental study. Also the social identities of the two recipients in these 10 items can be adjusted depending on the researcher's interests. For example, the recipients could be from two different real social (e.g., ethnic) groups. The method I describe here gives a clear quantitative estimate of average ingroup bias. Furthermore, it captures individual differences in the level of ingroup bias. These individual-level estimates can be outcome variables themselves. Alternatively, the researcher can use these estimates to predict an outcome variable of interest.

How much an average actor weights the outcomes of outgroups relative to ingroups affects important macro-level decisions. Barriers to international labor mobility (Rodrik, 2017), foreign assistance to poor countries (Kopczuk et al., 2005), redistributive tax policies in ethnically heterogeneous contexts (Rueda, 2018), intergroup trust and cooperation (Aksoy, 2015, Simpson, 2006), collective action in a competitive environment (Simpson and Aksoy, 2017) are among the many social outcomes that are directly influenced by the extent of ingroup favoritism. The current study shows that a relatively minor minimal group identity treatment with a highly educated subject pool (the majority of the subjects were Oxford University students) is enough to create strong levels of ingroup favoritism. One could easily imagine that the extent of ingroup favoritism would be higher when real groups and less selected subject pools are considered. It is thus not surprising to see widespread support to hostile policies toward foreigners in almost all countries. Furthermore, the current study shows that ingroup favoritism is much stronger when the outgroup is better off than the ingroup. This may explain why low income groups are more likely than high income groups to favor policies that reduce the outcomes of outgroups, such as "Brexit". This is because compared with high income groups, low income groups are more likely to be worse off than the outsiders.

Moreover, stressing that the outsiders are well educated and skilled may not change the opinion of the insiders. On the contrary, better off outsiders might trigger stronger ingroup favoritism from the insiders.

\section{References}

Aksoy, O., 2015. Effects of heterogeneity and homophily on cooperation. Social Psychology Quarterly 78 (4), 324-344.

Aksoy, O., Weesie, J., 2012. Beliefs about the social orientations of others: A parametric test of the triangle, false consensus, and cone hypotheses. Journal of Experimental Social Psychology 48 (1), 45-54.

Aksoy, O., Weesie, J., 2013. Hierarchical Bayesian analysis of biased beliefs and distributional social preferences. Games 4 (1), 66-88.

Aksoy, O., Weesie, J., 2014. Hierarchical bayesian analysis of outcome-and process-based social preferences and beliefs in dictator games and sequential prisoners dilemmas. Social Science Research $45,98-116$.

Becker, G. S., 1993. A treatise on the family. Cambridge, MA: Harvard University Press. 
Fischbacher, U., 2007. z-tree: Zurich toolbox for ready-made economic experiments. Experimental Economics 10 (2), 171-178.

Greiner, B., 2004. The online recruitment system ORSEE 2.0 a guide for the organization of experiments in economics. Working Paper Series in Economics 10, 1-15, mimeo, University of Cologne.

Griesinger, D. W., Livingston, J. J., 1977. Toward a model of interpersonal orientation in experimental games. Behavioral Science 18, 173-188.

Grzelak, J. L., Iwinski, T. B., Radzicki, J. J., 1977. Motivational components of utility. In: Jungermann, H., de Zeeuw, G. (Eds.), Decision making and change in human affairs. Dordrecht: Reidel.

Kopczuk, W., Slemrod, J., Yitzhaki, S., 2005. The limitations of decentralized world redistribution: An optimal taxation approach. European Economic Review 49 (4), 1051-1079.

Kuhlman, D. M., Brown, C., Teta, P., 1992. Judgements of cooperation and defection in social dilemmas: the moderating role of judge's social orientation. In: Liebrand, W., , Messick, D. M., Wilke, H. A. M. (Eds.), Social dilemmas, theoretical issues, and research findings. Oxford: Pergamon.

Liebrand, W., McClintock, C., 1988. The ring meaure of social values: a computerized procedure for assessing individual differences in information processing and social value orientation. Europoean Journal of Personality 2, 217-230.

Macro, D., Weesie, J., 2016. Inequalities between others do matter: evidence from multiplayer dictator games. Games 7 (2), 11.

McClintock, C. G., 1972. Social motivation - a set of propositions. Behavioral Science 31, 1-28.

Murphy, R. O., Ackermann, K. A., Handgraaf, M. J., 2011. Measuring social value orientation. Judgment and Decision Making 6 (8), 771-781.

Rabe-Hesketh, S., Skrondal, A., Pickles, A., 2002. Reliable estimation of generalized linear mixed models using adaptive quadrature. Stata Journal 2 (1), 1-21.

Rodrik, D., 2017. Is global equality the enemy of national equality? Harvard University working paper.

Rueda, D., 2018. Food comes first, then morals: Redistribution preferences, parochial altruism, and immigration in western europe. The Journal of Politics 80 (1), 225-239.

Schulz, U., May, T., 1989. The recording of social orientations with ranking and pair comparison procedures. European Journal of Social Psychology 19, 41-59.

Self, S. G., Liang, K.-Y., 1987. Asymptotic properties of maximum likelihood estimators and likelihood ratio tests under nonstandard conditions. Journal of the American Statistical Association $82,605-610$.

Sherif, M., Harvey, O. J., White, B. J., Hood, W. R., Sherif, C. W., et al., 1961. Intergroup conflict and cooperation: The Robbers Cave experiment. Vol. 10. University Book Exchange Norman, OK. 
Simpson, B., 2006. Social identity and cooperation in social dilemmas. Rationality and Society 18 (4), 443-470.

Simpson, B., Aksoy, O., 2017. Cumulative advantage in collective action groups: How competition for group members alters the provision of public goods. Social Science Research 66, 1-21.

Tajfel, H., 1970. Experiments in intergroup discrimination. Scientific American 223 (5), 96-102.

Tajfel, H., Billig, M. G., Bundy, R. P., Flament, C., 1971. Social categorization and intergroup behaviour. European Journal of Social Psychology 1 (2), 149-178.

Van Lange, P. A. M., 1999. The pursuit of joint outcomes and equaility of outcomes: an integrative model of social value orientation. Journal of Personality and Social Psychology 73, 337-349. 


\section{Appendix A. Other-other Decomposed Games}

Table A.2: 10 other-other Decomposed Games used in the study. The last three columns include percentages of subjects choosing option B in experimental and control groups and a t-statistics for the difference between the experimental and control groups, respectively ( $\mathrm{N}=146$ in the experimental group and $\mathrm{N}=40$ in the control group).

\begin{tabular}{|c|c|c|c|c|c|c|c|}
\hline \multirow[b]{3}{*}{ game } & \multicolumn{2}{|c|}{ Option A } & \multicolumn{2}{|c|}{ Option B } & \multicolumn{3}{|c|}{ Data } \\
\hline & (ingroup) & (outgroup) & (ingroup) & (outgroup) & $\% \mathrm{~B}$ & choices & difference \\
\hline & other gets & other gets & other gets & other gets & Exp. & Control & t-value \\
\hline 1 & 9 & 10 & 11 & 10 & .884 & .925 & 0.747 \\
\hline 2 & 10 & 9 & 10 & 11 & .651 & .900 & $3.124^{* *}$ \\
\hline 3 & 10 & 9 & 11 & 11 & .884 & .975 & $1.738^{+}$ \\
\hline 4 & 10 & 10 & 12 & 7 & .404 & .025 & $-4.794^{* * *}$ \\
\hline 5 & 10 & 10 & 15 & 6 & .616 & .300 & $-3.671^{* * *}$ \\
\hline 6 & 10 & 11 & 12 & 10 & .849 & .775 & -1.115 \\
\hline 7 & 10 & 14 & 12 & 8 & .575 & .025 & $-6.910^{* * *}$ \\
\hline 8 & 11 & 9 & 10 & 11 & .500 & .925 & $5.155^{* * *}$ \\
\hline 9 & 11 & 10 & 10 & 11 & .103 & .200 & $1.659^{+}$ \\
\hline 10 & 11 & 11 & 9 & 10 & .089 & .100 & 0.212 \\
\hline
\end{tabular}

